\title{
Quark correlations in the ground and excited nucleon states via the photo-absorption sum rules
}

\author{
Sergo Gerasimov ${ }^{1, *}$ \\ ${ }^{1}$ Joint Institute for Nuclear Research, Dubna, Russia
}

\begin{abstract}
The account of the dynamical correlations of quarks composing the nucleons is necessary for relevant description of nucleon and nuclear interactions. The present work is devoted to the use of the technique of known integral sum rules for the cross-sections and amplitude of the photo-hadron processes. In particular, a kind of the visual representation and quantification of the valence quark correlation characteristics referring to the photo-excitation of the nucleon resonances can be presented.
\end{abstract}

\section{Introduction: the past experience}

Instead of well-known non-relativistic dipole sum rules [1] for atomic and nuclear photoeffect,derived in the center-of-mass of the non-relativistic target systems:

$$
\sigma_{n}(E 1)=\int_{t h r}^{\infty} d \omega \omega^{n} \sigma_{E 1}(\omega)
$$

where: $n=-2$ refers to Kramers-Heisenberg sum rule (SR) for static electric-dipole polarizability of a given quantum system; $n=-1$ is the bremsstrahlung-weighted SR, depending of charged "parton" correlation in a given system; $n=0$ is the famous Thomas-Reiche-Kuhn SR, known as a precursor of the Quantum Mechanics itself, we should accept the picture of the transverse quark current densities of the fast moving nucleons. Just this framework gives fruitful and widely used approach for the description of the electromagnetic structure parameters of the nucleon.

In this paper, we return to the application of the current algebra technique in the infinite momentum frame used previously in the derivation of the Cabibbo-Radicati [2] sum rule and in the derivation of the earlier known from the dispersion theory [3-5] and current algebra [6] sum rules for the anomalous magnetic moments of the nucleon. In this way, a certain number of relations will be obtained directly depending on the flavor quark correlation in the ground nucleon state and in the states including an overlap of the ground and excited states of the nucleon.

\section{Sum rules with relativistic spin degrees of freedom}

In the relativistic theories the operators of electric dipole moments depend on the spin and following formally to the $p_{z} \rightarrow \infty$ techniques derivation of the Cabibbo-Radicati [2] or the

\footnotetext{
*e-mail: gerasb@theor.jinr.ru
} 
GDH s.r. derivation in the infinite momentum frame [6] we can derive the relations

$$
\begin{aligned}
& 4 \pi^{2} \alpha\left(\frac{1}{3}<\vec{D}^{2}>-\left(\frac{\kappa_{N}}{2 m_{N}}\right)^{2}\right)=\int \frac{d v}{v} \sigma_{t o t}^{r e s}(v), \\
& 4 \pi^{2} \alpha\left[\frac{1}{3}<\vec{D}^{2}>\right]=\int_{t h r}^{\infty} \frac{d v}{v}\left(\sigma_{p}^{r e s}(v),\right.
\end{aligned}
$$

where $\sigma_{p(a)}$ refers to the interaction cross section of the polarized nucleon and polarized photon with parallel (or anti-parallel) spins, $\kappa_{n}$-anomalous magnetic moment of the nucleon in the natural units,

$$
\hat{D}=\int \vec{x} \hat{\rho}(\vec{x}) d^{3} x=\sum_{j=1}^{3} Q_{q}(j) \vec{d}_{j} .
$$

The replacement of the constant $\kappa^{2}$ by its integral form illustrates and fulfills the essential element of the relationship and interdependence of the description of the static characteristics of a given system and known properties of its excited states.

The defined operators $Q_{q}(j)$ and $\vec{d}_{j}$ are the electric charges and configuration variables of point-like interacting quarks in the infinite-momentum frame of the bound system. Finally, we relate the electric dipole moment operator correlations, successively for the proton, the neutron and the pure "isovector-nucleon" part equal for both nucleons and the isovector part of the mean-squared radii operators entering the Cabibbo-Radicati sum rule, which all are sandwiched by the nucleon state vectors in the "infinite - momentum frame", with experimentally measurable data on the resonance parts of the photoabsorption cross sections on the proton and neutron presently known below $\sim 2 \mathrm{GeV}$. The listed operator mean values are parameterized as follows [7]

$$
\begin{aligned}
& R_{V}=\frac{1}{6}\left(<r_{1}^{2}>_{P}-<r_{1}^{2}>_{N}\right)=\frac{1}{3}\left(\alpha-\frac{1}{2} \beta\right), \\
& J_{P}=\frac{1}{3}<\hat{D}^{2}>_{P}=\frac{8}{27} \alpha+\frac{1}{27} \beta+\frac{8}{27} \gamma-\frac{8}{27} \delta, \\
& J_{N}=\frac{1}{3}<\hat{D}^{2}>_{N}=\frac{2}{27} \alpha+\frac{4}{27} \beta+\frac{2}{27} \gamma-\frac{8}{27} \delta, \\
& J_{V}=\frac{1}{3}<\hat{D}^{2}>_{V}=\frac{2}{12} \alpha+\frac{1}{12} \beta+\frac{2}{12} \gamma-\frac{4}{12} \delta,
\end{aligned}
$$

where $<{\overrightarrow{d_{1}}}^{2}>=<{\overrightarrow{d_{2}}}^{2}>=\alpha,<{\overrightarrow{d_{3}}}^{2}>=\beta,<\vec{d}_{1} \cdot \vec{d}_{2}>=\gamma,<\overrightarrow{d_{1}} \cdot \overrightarrow{d_{3}}>=<\overrightarrow{d_{2}} \cdot \overrightarrow{d_{3}}>=\delta$ and indices " 1 " and "2" refer to the like quarks, and " 3 " to the odd quark.

Evaluation of the relativistic electric dipole moment fluctuation was carried out with the available compilation [8] of the resonance pion-photoproduction data on the proton and neutron $A_{1 / 2}^{P(N)}$ and $A_{3 / 2}^{P(N)}$ the numerical values we calculated and all integrals over photoexcited nucleon resonances were taken in the narrow resonance approximation, when

$$
J_{p(a)}^{r e s} \simeq \frac{4 \pi m_{n}\left|A_{3 / 2(1 / 2)}^{\text {res }}\right|^{2}}{m_{\text {res }}^{2}-m_{n}^{2}},
$$

where $m_{n(r e s)}$ is the nucleon (or resonance) mass. Solving the system of the linear equations and evaluating the $R_{V}, J_{P, N, V}$ with the help of experimentally known partial amplitudes of main photo-excited resonances, we find our final results for the numerical values $\alpha, \beta$ and the opening angle $\theta_{12}$ and $\theta_{13}$ between vectors $\vec{d}_{1}$ and $\vec{d}_{2}$ and vectors $\vec{d}_{1}$ and $\overrightarrow{d_{3}}$, lying in the plane transverse to the "infinite momentum"' $p_{z} \rightarrow \infty$ vector [9]:

$$
\alpha^{1 / 2}=0.75 \pm 0.06 \mathrm{fm}, \beta^{1 / 2}=0.77 \pm 0.12 \mathrm{fm}, \theta_{12} \simeq 120^{0}, \quad \theta_{13} \simeq \theta_{12} \sim 120^{0} .
$$


We note that we could use the isoscalar input

$$
J_{S}=\frac{1}{3}<\hat{D}^{2}>_{S}=\frac{1}{108}(2 \alpha+\beta+2 \gamma+4 \delta)
$$

instead of the isovector one in Eq. (7). The numerical values of the parameters $\alpha, \beta, \gamma, \delta$ would turn out to be the same due to the use of the standard isotopic symmetry relations relating $J_{V}$ and $J_{S}$ with the $J_{P}$ and $J_{N}$.

Summarizing the results of the global approach via the use of the sum rules, we obtain a kind of averaged picture across the spectrum pattern with the presumably dominating influence of the first $\Delta(1236)$-resonance with its magnetic-dipole photo-excitation of the nucleon spin degrees of freedom, which is also hiding explication of the qualitative isospin-depending features of the (mainly, electric-dipole) 2-nd and (the electric-quadrupole) 3-rd resonance photo-excitation regions. The value of effective dipole moments, determining the excitation cross section of the "electric-dipole-type" nucleon resonances, includes apparently the pionic degrees of freedom of the constituent quarks. This is supported by the fundamental principle of the charge symmetry of strong interaction dynamics. The participation of the pion degrees of freedom plays the role in the dynamical formation of the quark-diquark spatially looking nucleon structure. We suggest to illustrate it numerically choosing the experimental data on the resonance photo-absorption data on the $J^{P}=3 / 2^{-}, P_{13}(1500)$ and $J^{P}=3 / 2^{-}, D_{33}(1600)$ resonances,composing the region of the "second" resonance area. Performing the evaluations analogous the earlier presented for all the resonances with the data from the PDG table [8], we obtain [9] the results differing from earlier cited

$$
\alpha^{1 / 2}=0.82 \mathrm{fm}, \beta^{1 / 2}=0.53 \mathrm{fm}, \theta_{12} \simeq 130^{0}, \theta_{13} \simeq 70^{0}, \theta_{23} \sim 160^{0} .
$$

A pronounced asymmetry of the sides of the triangles Oud and Ouu

$$
\frac{C_{u d}^{\min }}{C_{u u}} \simeq \frac{0.8 \mathrm{fm}}{1.5 \mathrm{fm}}
$$

can testify to the validity of the quark-diquark model of the considered resonances though diquarks are not apparently strongly bound. On the other hand, the obvious need to fulfill the charged symmetry requirements implies a significant role of the isospin-dependent quarkquark potentials, that is the role of the interactions caused by the pion exchanges, for instance. Ultimately, however, both factors can be the sides of the same phenomenon.

\section{References}

[1] J.S. Levinger, H.A. Bethe, Phys. Rev. 78, 115 (1950)

[2] N. Cabibbo, L.A. Radicati, Phys. Lett. 19, 678 (1966)

[3] S.B. Gerasimov, Sov. J. Nucl. Phys 2, 430 (1966)

[4] S.B. Gerasimov, Sov. J. Nucl. Phys 5, 1263 (1967)

[5] S.D. Drell, A.C. Hearn, Phys. Rev. Lett. 16, 908 (1966)

[6] M. Hosoda, K. Yamamoto, Prog. Theor. Phys. 36, 425 (1966)

[7] S.B. Gerasimov, Czech. J. Phys. 55, A209 (2005)

[8] K.A. Olive, et al. Chin. Phys. G 38, 090001 (2014)

[9] S. B. Gerasimov, J. Phys.: Conf. Ser. 678012009 (2016) 